\title{
Pre-conceptual study of the European DEMO neutron diagnostics
}

\author{
M. Cecconello, ${ }^{a, 1}$ S. Conroy, ${ }^{a}$ G. Ericsson, ${ }^{a}$ H. Hjalmarsson, ${ }^{a}$ T. Franke, ${ }^{b}$ and W. Biel ${ }^{c, 2}$ \\ ${ }^{a}$ Department of Physics and Astronomy, Uppsala University, EURATOM-VR Association, Uppsala, Sweden \\ ${ }^{b}$ Max-Planck-Institut für Plasmaphysik and EUROfusion, Power Plant Physics and Technology Department, \\ Garching, Germany \\ ${ }^{c}$ Institute of Energy and Climate Research, Forschungszentrum Jülich GmbH, Germany \\ E-mail: marco.cecconello@physics.uu.se
}

\begin{abstract}
This paper discusses the pre-conceptual design of the European DEMO neutron diagnostics aimed at the measurement of the fusion power, the plasma displacement and the fuel ion ratio for plasma burn control. The design has been based on the European DEMO design concept with a single divertor and on the so-called DEMO1 plasma scenario but limited only to the steady phased of the discharge. The suggested neutron diagnostics consist of neutron activation systems, fission chambers and vertical and horizontal collimated neutron flux monitors with spectroscopy capabilities. The fusion power can be measured with an accuracy of $1 \%$ with a time resolution of one tenth of the energy confinement time and the plasma displacement with a time resolution of 1 ms. Central ion temperature and fuel ion ratio instead can be measured with a time resolution of about $1 \mathrm{~s}$. Calibration and interfacing issues are discussed.
\end{abstract}

Keywords: Neutron detectors, Fast neutrons, Nuclear instruments and methods for hot plasma diagnostics.

${ }^{1}$ Corresponding author.

${ }^{2}$ Also at Department of Applied Physics, Ghent University, Belgium 


\section{Contents}

1 Introduction 1

2 Neutron Diagnostics 2

2.1 Collimated neutron flux monitors 2

2.2 Uncollimated fission chambers 5

2.3 Neutron activation system 5

2.4 Neutron detectors 5

3 Calibration 6

4 Interfacing issues $\quad 6$

$\begin{array}{llr}5 & \text { Future developments } & 8\end{array}$

\section{Introduction}

A demonstration fusion power plant (DEMO) is the only step between ITER and a commercial power plant according to the European roadmap. The main goals of DEMO are to achieve tritium self-sufficiency and to deliver significant net electricity to the grid. The extremely harsh conditions in DEMO, especially the high neutron fluxes and fluences, coupled with the requirement of a Tritium Breeding Ratio (TBR) > 1 resulting in the limited views of the plasma pose unprecedented challenges to the diagnostics necessary to operate DEMO safely. In addition, the diagnostics, whose main aim will be plasma control, should have high accuracy, availability by redundancy and reliability for long period of times (full power years). These considerations limit the number and scope of the diagnostics on DEMO. The reference plasma scenario, extrapolated from ITER ELMy $\mathrm{H}$-mode, for the pre-conceptual study of the neutron diagnostics is the so-called DEMO1 [1-3] characterized by pulsed operation and, during the steady-state phase, by a fusion power $P_{\mathrm{f}} \approx 2$ $\mathrm{GW}$, core temperatures $T_{\mathrm{i}, \mathrm{e}} \approx 25 \mathrm{keV}$ and densities $n_{\mathrm{i}, \mathrm{e}} \approx 1 \times 10^{20} \mathrm{~m}^{-3}, Z_{\mathrm{eff}} \approx 2.2$ and with an energy confinement time $\tau_{\mathrm{E}} \approx 4 \mathrm{~s}$. The resulting neutron emission is dominated by the thermal emission of $14.1 \mathrm{MeV}$ neutron from the DT fuel ions with a yield $Y_{\mathrm{n}} \approx 7 \times 10^{20} \mathrm{~s}^{-1}$ and a peak core neutron emissivity $\varepsilon \approx 10^{18} \mathrm{~s}^{-1} \mathrm{~m}^{-3}$. The reference baseline tokamak design used in this study is based on a lower single null divertor with access to the plasma through large divertor, horizontal and vertical ports [4]. However, the TBR $>1$ requirement imposes strong constraints on the total footprint area of diagnostics openings on the tritium Breeding Blanket (BB). The design of DEMO is not finalized and significant deviations from the current one will require a substantial redesign of the neutron diagnostics presented in this work and assessment of their feasibility and performances. Pre-conceptual design of DEMO diagnostics is, at this stage, limited only to the steady state phase of the DEMO1 plasma scenario: ramp-up and ramp-down phases will be addressed in a future study. 


\section{Neutron Diagnostics}

Neutron Diagnostics (NDs) are intended to contribute to the three main aspects of plasma burn control: $i$ ) equilibrium control by measuring the plasma position and shape, ii) kinetic control by measuring $P_{\mathrm{f}}$ and the fuel ion ratio and iii) instability detection by measuring the neutron yield fluctuations [5]. Generic physics requirements for burn control are that the fusion power $P_{\mathrm{f}}$ should be controlled to keep the variations of fusion power always below $\pm 20 \%$ over not more than 20 seconds, that plasma displacements $D<15 \mathrm{~cm}$ should be recoverable and that MHD events should be detectable on a time scale faster than $0.1 \mathrm{~s}$. However, at this stage of DEMO development, no specific measurement requirements have been elaborated for the plasma parameters needed for burn control in terms of spatial and temporal resolution, accuracy and precision. As a result, this pre-conceptual design has been based on typical requirements that are placed on NDs in present day tokamaks and particularly in ITER. Measurement requirements for DEMO will be elaborated in the next phase of the design of the plasma control system. The measurement techniques and detectors envisaged to provide the required inputs to the control actuators are listed in table 1 and are based on well-known and mature methods that are implemented in all large tokamaks such as JET and under design for ITER. The main ND system on DEMO consists of two collimated neutron flux (yield) monitors (Neutron Cameras, NCs) equipped with Fission Chambers (FCs) and Diamond Detectors (DDs) which will provide the neutron emissivity profile $\varepsilon$ from which the fusion power $P_{\mathrm{f}}$ and the plasma displacement $D$ can be estimated. The DDs, thanks to their neutron spectroscopic capabilities, will provide the fuel ion ratio and the ion temperature in the core. Uncollimated FCs and micro-FCs ( $\mu \mathrm{FCs}$ ), discussed in section 2.2, can in principle provide the same information as the collimated neutron flux monitors but have no spectroscopic capability. Finally, Neutron Activation Systems (NASs) will provide the NDs absolute calibration and are briefly discussed in section 2.3 but, due to their slower response time, are not directly used for the plasma real time control.

\begin{tabular}{|c|c|c|c|}
\hline Control & Parameter & Method & Detectors \\
\hline Equilibrium & Displacement & $\begin{array}{l}\text { 2D profile monitors } \\
\text { Total neutron yield }\end{array}$ & $\begin{array}{l}\text { FC, Diamonds } \\
\mu \mathrm{FC}\end{array}$ \\
\hline Kinetic & Fusion power & $\begin{array}{l}\text { 2D profile monitors } \\
\text { Total neutron yield }\end{array}$ & $\begin{array}{l}\text { FC, Diamonds } \\
\mu \mathrm{FC}\end{array}$ \\
\hline & Fuel ion ratio & Spectroscopy & Diamonds \\
\hline & Core ion temperature & Spectroscopy & Diamonds \\
\hline MHD & Yield fluctuations & $\begin{array}{l}\text { 2D profile monitors } \\
\text { Total neutron yield }\end{array}$ & $\begin{array}{l}\text { FC, Diamonds } \\
\mu \mathrm{FC}\end{array}$ \\
\hline
\end{tabular}

Table 1. Neutron measurements methods foreseen in DEMO for plasma control.

\subsection{Collimated neutron flux monitors}

The design guiding principles for the NCs have been: $i$ ) collimator diameter as small as possible but compatible with statistical uncertainties in the counts above threshold $<1 \%$; ii) sufficient number of Lines of Sight (LoS) for accurate reconstruction of $\varepsilon$ and $P_{\mathrm{f}}$; iii) vertical and horizontal views for plasma position control and $\varepsilon$ reconstruction via real time tomography inversion; $i v$ ) LoS geometry 
to cover a as large as possible area of the neutron emissivity region and being compatible with the constraints posed by the port plug; $v$ ) detectors located outside the bio-shield and $v i$ ) with energy threshold and spectroscopic capabilities. The integration of the Horizontal and Vertical Neutron Cameras (HNC and VNC respectively) LoS are shown in the left panel of figure 1. Two collimator
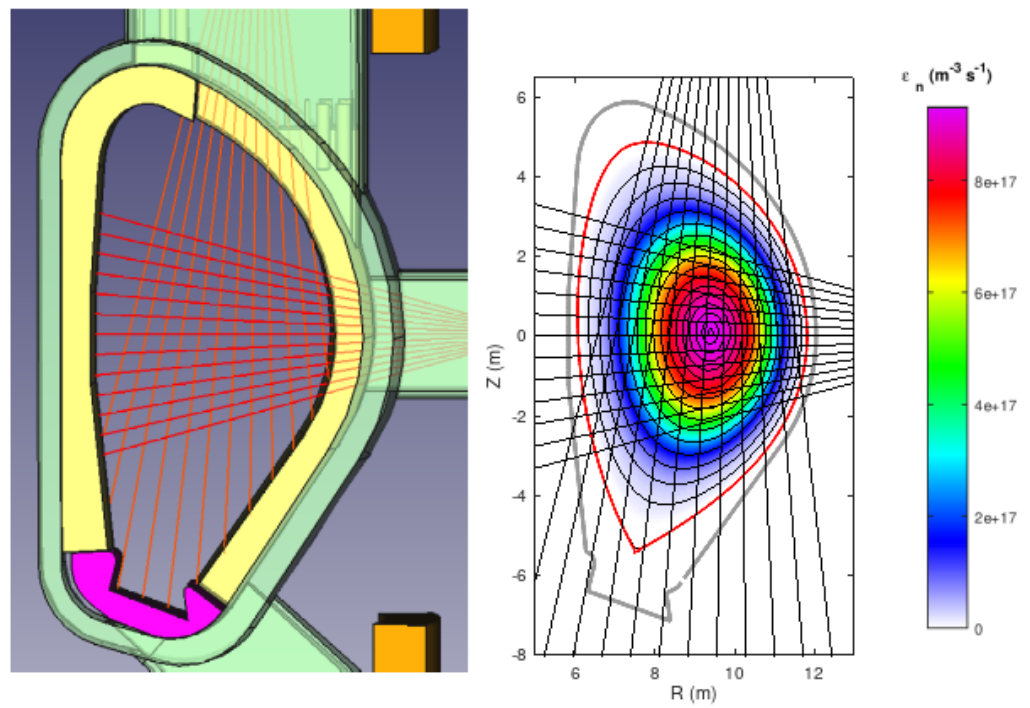

Figure 1. Left panel: HNC and VNC LoS integrated in the DEMO 2017 baseline design: blanket module in yellow, divertor in magenta and vacuum vessel in green. Right panel: neutron emissivity for the DEMO1 plasma scenario with the HNC and VNC LoS superimposed. LoS numbering: HNC 1 to 13 from top to bottom, VNC 14 to 25 from outboard to inboard.

solutions have been optimized, one to provide $P_{\mathrm{f}}$ with a time resolution of $\tau_{\mathrm{E}} / 10=0.4 \mathrm{~s}, n_{\mathrm{D}} / n_{\mathrm{T}}$ with a time resolution of a tenth of the particle confinement time $\left(\tau_{\mathrm{P}} / 10=1.6 \mathrm{~s}\right)$ and the other to measure the $D$ with a time resolution of $1 \mathrm{~ms}$. In both cases, collimator lengths of $\approx 10 \mathrm{~m}$ for the $\mathrm{HNC}$ and between 15 and $17 \mathrm{~m}$ for the VNC are required to position the detectors outside the bioshield. In the first case, the collimator diameters were adjusted to achieve a count rate of $2.5 \times 10^{4}$ $\mathrm{s}^{-1}$ across all channels corresponding to $10^{4}$ counts for the integration time $\tau_{\mathrm{E}} / 10$ and assuming an efficiency of $10^{-4}$ which is typical for FCs. In this configuration, the collimator diameters varies in the range 1.1 to $1.4 \mathrm{~cm}$ for the $\mathrm{HNC}$ and 1.3 to $3.6 \mathrm{~cm}$ for the $\mathrm{VNC}$ with an overall footprint area on the BB surface of $10^{-3} \mathrm{~m}^{2}$, the accuracy in the reconstruction of $\varepsilon$ and $P_{\mathrm{f}}$ is better than $10 \%$ up to 0.85 of the normalized poloidal flux coordinate and $1 \%$ respectively. The fluence at the detector location over DEMO life-time (assuming 6 full-power years) is in the range $0.05-2.00 \times 10^{20}$ $\mathrm{m}^{-2}$. For comparison, in ITER, estimates of the fluences at the end of the collimators of the radial neutron camera vary in the range of $0.3-3.0 \times 10^{20} \mathrm{~m}^{-2}$ and thus not expected to be an issue for FCs in DEMO. The impact on DDs is being investigated at neutron irradiation test facilities and it is reasonable to assume that it will be known well ahead of the conceptual design phase. A time resolution of $1 \mathrm{~ms}$ for real time plasma control position requires larger collimator diameters $(\geq 3$ $\mathrm{cm})$ to compensate for the reduced integration time if the same statistical uncertainty is required. In this case, the overall footprint area of the NCs is $7 \times 10^{-2} \mathrm{~m}^{2}$ and the fluence is increased to $2.2 \times 10^{21} \mathrm{~m}^{-2}$. Relaxing the time resolution to $10 \mathrm{~ms}$ and accepting a statistical uncertainty on 
the counts of $5 \%$ would allow to maintain the same collimator diameters used for fusion power measurements. The horizontal and vertical plasma displacement from the reference equilibrium position is inferred by the normalized asymmetry $A$ between the counts in the two halves of the VNC and HNC respectively:

$$
A=\frac{\sum_{i=1}^{M} n_{i}-\sum_{i=M+1}^{N} n_{i}}{\sum_{i=1}^{N} n_{i}},
$$

where $n_{i}$ indicates the counts in the $i$-th $\operatorname{LoS}, N$ the total number of $\operatorname{LoS}$ and $M$ is the nearest integer to $N / 2$. The left panel of figure 2 shows the linear dependence of $A$ on the rigid horizontal and vertical displacements $D$ of the plasma column: the sensitivity $A / D$ is 0.32 and $1.2 \mathrm{~m}^{-1}$ for the VNC and $\mathrm{HNC}$ respectively. The uncertainty $\delta D$ in the plasma vertical and horizontal displacement, for large collimator diameters with an integration time of $1 \mathrm{~ms}$ and considering as uncertainty in $A$ only counting statistics, is $\delta D= \pm 3 \mathrm{~cm}$ : this could be reduced to $\delta D= \pm 1 \mathrm{~cm}$ by increasing the integration time to $10 \mathrm{~ms}$.
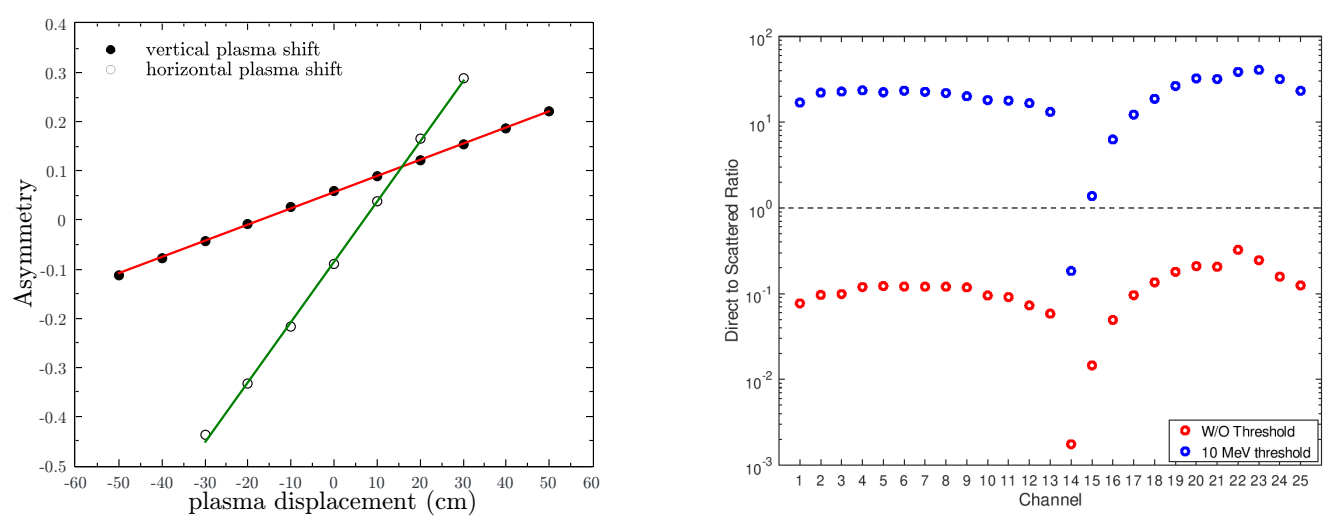

Figure 2. Left panel: asymmetry as a function of the plasma displacement. Right panel: diamond direct to scattered neutron ratio: channels 1 to 13 (HNC) and 14 to 25 (VNC).

Monte Carlo particle transport codes have been used to estimate the ratio between direct and scattered neutrons at the location of the DDs: the results, shown in the right panel of figure 2, indicate that this ratio is $>10$ for most $\mathrm{LoS}$ when a $10 \mathrm{MeV}$ energy threshold is applied. Estimates of the ion core temperature and on the fuel ion ratio will be carried out using the spectroscopic capabilities of the DDs. In particular the latter is based on the measurements of neutrons from thermal and supra-thermal DT reactions [6]. This method, successfully demonstrated at JET in the range $0.1<n_{\mathrm{T}} / n_{\mathrm{D}}<10$, requires finding the beam/plasma emission fraction (estimated to be $\approx 2 \%$ in DEMO) with a maximum $10 \%$ statistical error which would limit this measurements to $\mathrm{HNC} / \mathrm{VNC}$ central channels and to a time resolution of approximately $1 \mathrm{~s}$. For redundancy consideration, a second set of identical $\mathrm{HNC}$ and VNC is planned to be installed at another toroidal location. 


\subsection{Uncollimated fission chambers}

Absolutely calibrated FCs provide the time resolved neutron flux with a time resolution on the ms level thus enabling this diagnostic to be used for real time control of $P_{\mathrm{f}}$. A tentative proposal for DEMO consists of 12 FCs ( 8 active and 4 blanks, used for $\gamma$-rays background subtraction) located in the equatorial plane at four different toroidal sections. The exact position will be determined in a future work. The FCs are divided in two subgroups each one consisting of four active FCs and two blanks with different sensitivities spanning the neutron yield range from $10^{11} \mathrm{~s}^{-1}$ to $10^{2} \mathrm{~s}^{-1}$ also thanks to the possibility to operate them in different modes (pulse counting, Campbelling and current mode). In addition, a set of two $\mu$-FCs, placed above and below the mid plane, could be used for the control of the plasma vertical position on the ms time scale. The choice and amount of fissile/fissionable material is not defined at this stage: ${ }^{238} \mathrm{U}$ is considered a good candidate due to its fission energy threshold of few MeVs which allows the suppression of scattered neutrons. However, breeding of fissile ${ }^{239} \mathrm{Pu}$ through radiative capture of thermal neutrons is a concern due to the expected high neutron fluencies of up to $10^{25} \mathrm{~m}^{-2}$ behind the BB modules and can, over time, significantly change the FCs response characteristics. This however is not considered an issue as long as they can be cross-calibrated against the activation foils. A possible alternative to be investigated in the near future is the use of ${ }^{242} \mathrm{Pu}$ instead.

\subsection{Neutron activation system}

Neutron activation systems based on foils are used at most high performance fusion devices such as JET [7], KSTAR [8] and JT-60U [9]. ITER has planned a NAS with a total of 12 different irradiation positions [10]. NASs can provide an absolutely calibrated measurement of the local neutron fluence, provided the neutron energy spectrum at the irradiation location is known, from which $P_{\mathrm{f}}$ can be estimated. For this, modelling of the neutron transport is essential as well as knowledge of the neutron emissivity profile. In addition, the irradiation position should be as close to the plasma as practically possible and, to make NASs reasonably insensitive to shifts in the plasma position, several poloidal positions should be considered. A minimal NAS for DEMO composed of 6 irradiation positions, divided into three poloidal positions (inboard, outboard upper and outboard lower) in two different toroidal sections has been considered for the calibration of the NDs and for $P_{\mathrm{f}}$ measurement on a slower time scale than the one needed for real time control. Activation materials such as ${ }^{115} \mathrm{In}$ and ${ }^{93} \mathrm{Nb}$ are suitable for short- to medium-term exposures of $\mathrm{D}$ and DT plasmas, ${ }^{59} \mathrm{Co}$ is more suitable for long-term exposures for dose and neutron history purposes. In this context, it should also be noted that the measurement of activation of water in dedicated loops has been proposed for ITER [11]. Future developments require an investigations of the number and poloidal and toroidal location of the irradiation stations in order to assess their sensitivity to variations in plasma position, how deep they should be embedded in the BB modules and how the capsule transfer lines interface with the BB. Furthermore, an assessment of detailed design and material choices for the transfer capsules is needed.

\subsection{Neutron detectors}

Potential detectors for the $\mathrm{VNC} / \mathrm{HNC}$ have been investigated by taking into account their counting and neutron spectroscopic capabilities, radiation hardness, temperature resistance, magnetic field 
compatibility and time response. Among the considered detectors are commercially available DDs, FCs, GEM, MICROMEGAS and He4 detectors. He4 detectors have been excluded due to the slow scintillation events (and therefore slow time response). GEM detectors are limited to operate below $50{ }^{\circ} \mathrm{C}$ and have no spectroscopic capabilities. At this stage, ${ }^{238} \mathrm{U}$ FCs and DDs are the best option although the radiation hardness of the latter has not yet been fully confirmed [12]. Of possible interest are detectors based on depleted ${ }^{238} \mathrm{UO}_{2}$ thanks to their capabilities of operating at high temperatures (> 2600 K) and greater radiation and electromagnetic noise resistance [13]. The major drawbacks is their ${ }^{235} \mathrm{U}$ content and the fission products activation of detectors themselves. Detectors based on ${ }^{238} \mathrm{UO}_{2}$ are at an early stage and extensive research and development will be required to translate these first study tests into a prototype detector. Maintenance of detectors outside the bio-shield is not deemed to be more complicated nor more difficult than that planned in ITER for areas with similar shut-down dose rate. The detectors will nonetheless be activated and therefore remote handling will be required.

\section{Calibration}

The absolute calibration of the FCs and of the DDs in the HNC and VNC is based on the ITER Radial Neutron Camera (RNC) calibration strategy [14]. Before their installation, the detectors response function, efficiency and, in the case of DDs, energy resolution should be characterized in the neutron energy $E_{\mathrm{n}}$ range $2-20 \mathrm{MeV}$ and at $E_{\mathrm{n}}=2.45$ and $E_{\mathrm{n}}=14 \mathrm{MeV}$ neutron energies using different neutron and $\alpha$-particles source. The FCs efficiency to 2.5 and $14 \mathrm{MeV}$ neutrons should be determined to reduce the inevitable variation of the fissionable layer thickness in each FC . Diamond detectors energy calibration with a relative uncertainty less than $1 \%$ should be carried out using at least three different $\alpha$-particles energies (for example using a mix of ${ }^{239} \mathrm{Pu},{ }^{241} \mathrm{Am},{ }^{244} \mathrm{Cm}$ $\alpha$-sources). The neutron detection efficiency should be determined with a relative uncertainty below $5 \%$ thus imposing a $2 \%$ maximum limit in the relative uncertainty in the ${ }^{12} \mathrm{C}(\mathrm{n}, \alpha){ }^{9} \mathrm{Be}$ peak counting statistics. Once installed on DEMO, the absolute calibration of FCs and DDs will be determined by cross-referencing their response to reference plasma discharges with the NAS by means of neutron transport Monte Carlo simulations. Cross-calibration against the NASs will be done with reference plasma discharges complemented by neutron transport calculations. Long term monitoring of the calibration is required and could be carried out using a combination of embedded neutron and $\alpha$-particle sources together with reference plasmas discharges. As in ITER and for the same motivations [14], no in-vessel calibration with a strong ${ }^{252} \mathrm{Cf}$ or DD/DT neutron generator is foreseen. The HNC and VNC detectors, being located outside the bio-shield, have potentially more space available for the installation of small DD/DT neutron generator which could be used for their long term calibration without having to recur to dedicated plasma discharges in DEMO. DD/DT neutron generators however would need to be themselves constantly monitored [15].

\section{Interfacing issues}

Dedicated slim diagnostic cassettes in between BB segments will house the HNC and VNC collimators thus avoiding impacting the internal structure of the BBs. For this reason, the NASs and uncollimated $\mu$-FCs should also be mounted inside the slim cassette together with the necessary 
pipes and cabling. This is a critical design interfacing issue that needs to be addressed as soon as possible as otherwise the integration of NASs and $\mu$-FCs will have a major impact on the internal structure of the BBs. The HNC and VNC interface with the vacuum vessel is via the collimator openings which will be sealed by multiple thin flanges whose impact on the direct neutron flux can be easily modelled with Monte Carlo neutron transport codes. A study of the interface between the collimators and their shielding $\left(\mathrm{B}_{4} \mathrm{C}\right)$ has highlighted some issues with the port plug structures, the cryostat, the bio-shielding and DEMO building as shown in figure 3 which affect mainly the outermost channels of the HNC. These will be addressed in a future revision this pre-conceptual design together with a more detailed study of the location of the fission chambers and activation system. The long collimators should be divided into separate segments to allow for the mechanical movement between vacuum vessel, BBs, port plugs and bio-shield: these will introduce a misalignment between collimator segments which can cause a reduction of the neutron flux at the detector. An initial investigation showed that a radial misalignment of $4 \mathrm{~cm}$ (between a collimator front and back sections, each of $2 \mathrm{~m}$ length and $2 \mathrm{~cm}$ diameter, separated by a distance of $8 \mathrm{~m}$ ) will cause an effective loss of neutron flux of approximately $10 \%$ suggesting that quite large misalignments can be coped with using this type of collimation design. The shielding of the detectors located outside the bio-shield will mainly interface with the building structures and future work will focus on possible solutions which might impact the geometry of the LoS, the building structures or both.

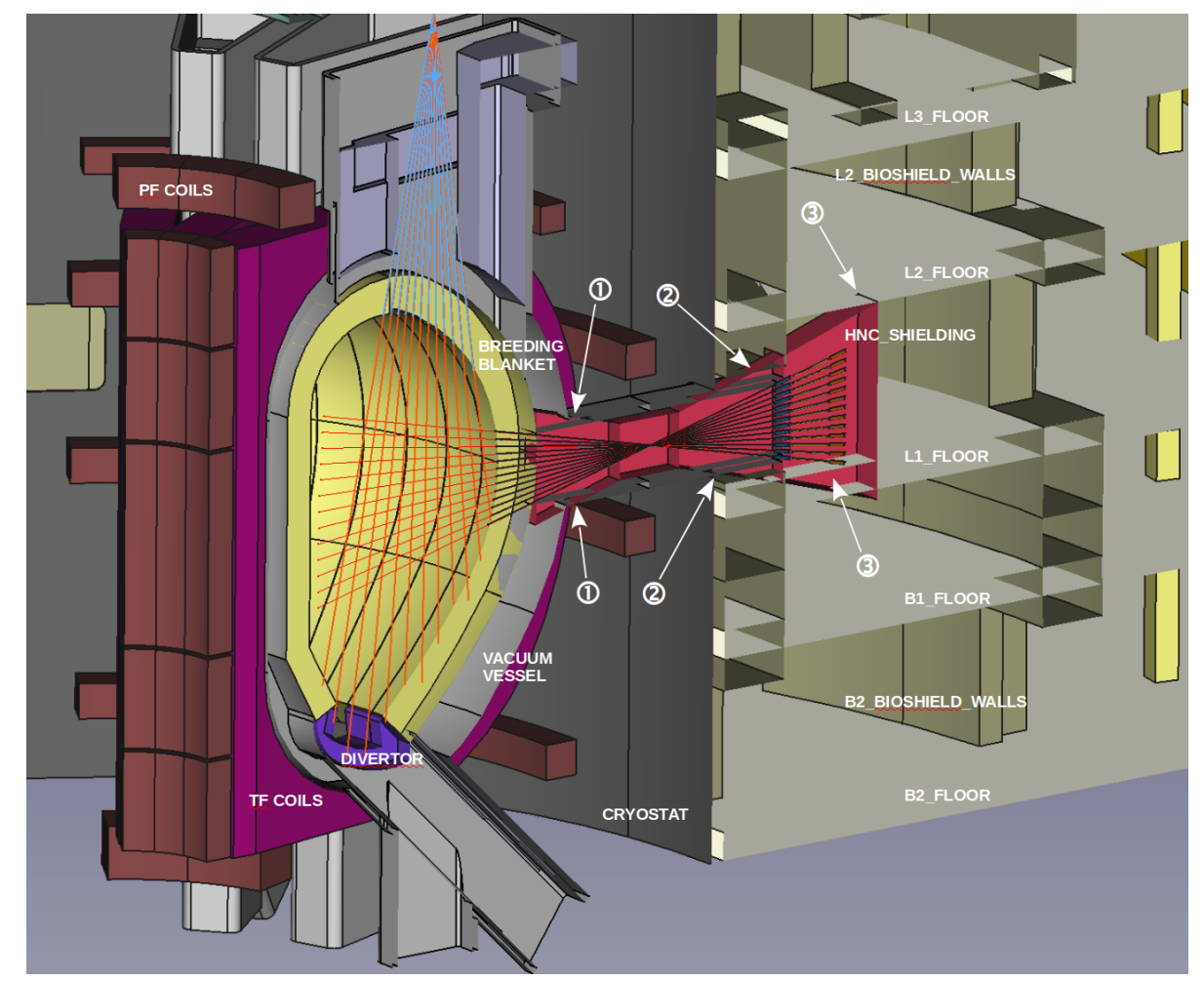

Figure 3. HNC shielding (dark red) interfacing issues with DEMO vacuum vessel (1), the cryostat and the bioshield walls (2) and with the building floors (3). 


\section{Future developments}

Several activities are required to bring this pre-conceptual design to the maturity level required for a conceptual design. Among these are: $i$ ) the integration of NASs and $\mu$-FCs and of their ancillary equipment within the slim diagnostic cassette, $i i$ ) the redesign of the geometry of the lines of sight for integration in the DEMO building and port plug, iii) a detailed design of the neutron shielding in the port plug and around the detectors outside the bio-shield, $i v$ ) the calculation of the shut-down dose rate outside the bio-shield and $v$ ) the identification of activation and fissile/fissionable materials. The feasibility of controlling the plasma position using collimated neutron flux monitors should be tested in presented day tokamaks such as JET, where a real-time data acquisition system for the neutron camera is already available, and in the future in the Divertor Test Tokamak. Compatibility of diamond detectors with the large neutron fluences expected in DEMO should be verified for example by exploiting similar tests to those carried out for the neutron diagnostics in ITER. Finally, the design of the neutron diagnostic should be extended to include the ramp-up and ramp-down phases of the plasma discharge.

\section{Acknowledgments}

This work has been carried out within the framework of the EUROfusion Consortium and has received funding from the Euratom research and training programme 2014-2018 and 2019-2020 under grant agreement No 633053. The views and opinions expressed herein do not necessarily reflect those of the European Commission. This work has been supported by Vetenskaprådet reserach grant no. 2015-03869.

\section{References}

[1] Federici G, Kemp R, Ward D, Bachmann C, Franke T, Gonzalez S, et al. Overview of EU DEMO design and $R \& D$ activities. Fusion Engineering and Design. 2014, 89, 882

[2] Federici G, Bachmann C, Barucca L, Biel W, Boccaccini L, Brown R, et al. DEMO design activity in Europe: Progress and updates. Fusion Engineering and Design 2018, 136, 729

[3] Giruzzi G, Artaud JF, Baruzzo M, Bolzonella T, Fable E, Garzotti L, et al. Modelling of pulsed and steady-state DEMO scenarios. Nucl Fusion 2015, 55(7), 073002

[4] Federici G, Bachmann C, Barucca L, Baylard C, Biel W, Boccaccini LV, et al. Overview of the DEMO staged design approach in Europe. Nucl Fusion 2019, 59(6), 066013

[5] Biel W Diagnostics for plasma control - From ITER to DEMO, Fusion Eng. Design. in press (2019) https://doi.org/10.1016/j. fusengdes.2018.12.092

[6] Hellesen C et al. Prospects for measuring the fuel ion ratio in burning ITER plasmas using a DT neutron emission spectrometer, Rev. of Sci. Instr. 85, 11D825, 2014

[7] Bertalot L et al. Calibration of the JET neutron activation system for DT operation. Review of scientific instruments. 1999, 70(1), 1137-1140.

[8] Cheon M S et al. Diagnostic neutron activation system for KSTAR. J Inst. 2012, 7(05), C05009-C05009. 
[9] Hoek Met al. Triton burnup measurements by neutron activation at JT-60U. Nuclear Instruments and Methods in Physics Research Section A: Accelerators, Spectrometers, Detectors and Associated Equipment. 1996, 368(3), 804-814.

[10] Krasilnikov V et al. Neutron Activation System for ITER Tokamak. In: Alghem Hamidatou L, editor. Advanced Technologies and Applications of Neutron Activation Analysis. IntechOpen; 2019

[11] Nishitani T et al. Neutron activation system using water flow for ITER. Review of Scientific Instruments. 2003, 74(3), 1735-8.

[12] Baccaro S, Cemmi A, Di Sarcina I, Esposito B, Ferrara G, Grossi A, et al. Radiation Damage Tests on Diamond and Scintillation Detector Components for the ITER Radial Neutron Camera, IEEE Trans Nucl Sci. 2018, 65(8), 2046-53.

[13] Thomas Meek Semiconductive properties of UO2, Conf. On Waste Management symposium 2001, Tucson Arizona.

[14] Cecconello M, Miklaszewski R, Marocco D, Conroy S, Moro F, Esposito B, et al. Strategy and guidelines for the calibration of the ITER Radial Neutron Camera, Fusion Eng. Design. 2019 S0920379619304272

[15] Batistoni P, Popovichev S, Cufar A, Ghani Z, Giacomelli L, Jednorog S, et al. 14 MeV calibration of JET neutron detectors-phase 1: calibration and characterization of the neutron source, Nuclear Fusion 2018, 58(2), 026012. 